Bull. Korean Math. Soc. 49 (2012), No. 3, pp. 609-619

http://dx.doi.org/10.4134/BKMS.2012.49.3.609

\title{
FOURIER-TYPE FUNCTIONALS ON WIENER SPACE
}

\author{
Hyun Soo Chung and Vu Kim Tuan
}

\begin{abstract}
In this paper we define the Fourier-type functionals via the Fourier transform on Wiener space. We investigate some properties of the Fourier-type functionals. Finally, we establish integral transform of the Fourier-type functionals which also can be expressed by other Fouriertype functionals.
\end{abstract}

\section{Introduction}

Let $\hat{f}$ be the Fourier transform of $f$,

$$
\hat{f}(\vec{\xi})=\left(\frac{1}{2 \pi}\right)^{\frac{n}{2}} \int_{\mathbb{R}^{n}} f(\vec{u}) \exp \{i \vec{u} \cdot \vec{\xi}\} d \vec{u}, \quad \vec{\xi}, \vec{u} \in \mathbb{R}^{n},
$$

where $\vec{u} \cdot \vec{\xi}=u_{1} \xi_{1}+\cdots+u_{n} \xi_{n}$. By the spectrum of $f$ (or the support of $\hat{f}$ ), we mean the smallest closed set outside which $\hat{f}$ vanishes almost everywhere. The Fourier transform is a very powerful mathematical object. The classical Paley-Wiener theorem [10] describes functions of compact spectrum through its analytic extension into the complex space and its growth at infinity. In $[1,11]$ functions with compact spectrum were described through the norms of differential operators of infinite order. So by a Paley-Wiener-type theorem we understand any result describing functions with spectrum of a given configuration.

Let $C_{0}[0, T]$ denote one-parameter Wiener space; that is the space of continuous real-valued functions $x$ on $[0, T]$ with $x(0)=0$. Let $\mathcal{M}$ denote the class of all Wiener measurable subsets of $C_{0}[0, T]$, and let $m$ denote Wiener measure. $\left(C_{0}[0, T], \mathcal{M}, m\right)$ is a complete measure space, and we denote the Wiener integral of a Wiener integrable functional $F$ by

$$
\int_{C_{0}[0, T]} F(x) d m(x) .
$$

Received March 1, 2011; Revised June 10, 2011.

2010 Mathematics Subject Classification. Primary 42B10, 28C20.

Key words and phrases. integral transform, Fourier transform, Fourier-type functional, Wiener space, Paley-Wiener theorem. 
A subset $B$ of $C_{0}[0, T]$ is said to be scale-invariant measurable provided $\rho B$ is $\mathcal{M}$-measurable for all $\rho \geq 0$, and a scale-invariant measurable set $N$ is said to be a scale-invariant null set provided $m(\rho N)=0$ for all $\rho \geq 0$. A property that holds except on a scale-invariant null set is said to hold scale-invariant almost everywhere (s-a.e.) [4]. Throughout this paper we will assume that each functional $F: C_{0}[0, T] \rightarrow \mathbb{C}$ that we consider is scale-invariant measurable and that

$$
\int_{C_{0}[0, T]}|F(\rho x)| d m(x)<\infty
$$

for each $\rho>0$.

In a unifying paper [6], Lee defined an integral transform $\mathcal{F}_{\gamma, \beta}$ of analytic functionals on abstract Wiener spaces. In [5], Kim and Skoug gave a necessary and sufficient condition that a functional $F$ in $L_{2}\left(C_{0}[0, T]\right)$ has an integral transform $\mathcal{F}_{\gamma, \beta} F$ also belonging to $L_{2}\left(C_{0}[0, T]\right)$. In [2] the authors established several very basic formulas relating convolution products, integral transforms and inverse integral transforms for functionals in $L_{2}\left(C_{0}[0, T]\right)$. Also see paper [3], for further work involving generalized integral transforms and convolutions.

In this paper, we define the Fourier-type functionals via the Fourier transform on a function space and investigate some properties of the Fourier-type functionals. Finally, we establish integral transform of the Fourier-type functional which also can be expressed by other Fourier-type functionals. The proofs are based on the Parseval equality and some properties of the Fourier transform on $\mathbb{R}^{n}$.

\section{Definitions and preliminaries}

In this section we state some definitions and preliminaries used in this paper.

Let $K=K_{0}[0, T]$ be the space of all complex-valued continuous functions defined on $[0, T]$ which vanish at $t=0$. First we recall the definition of the integral transform $\mathcal{F}_{\gamma, \beta}$ introduced in [6] and used in $[2,5]$.

Definition 2.1. Let $F$ be a functional defined on $K$. For each pair of nonzero complex numbers $\gamma$ and $\beta$, the integral transform $\mathcal{F}_{\gamma, \beta} F$ of $F$ is defined by

$$
\mathcal{F}_{\gamma, \beta}(F)(y) \equiv \int_{C_{0}[0, T]} F(\gamma x+\beta y) d m(x), \quad y \in K,
$$

if it exists.

Now we state some well-known properties of the Fourier transform as a lemma.

Lemma 2.2. (1) Let $f \in C^{\infty}\left(\mathbb{R}^{n}\right)$ be such that all the Laplacians $\Delta^{k} f, k=$ $0,1, \ldots$, belong to $L_{2}\left(\mathbb{R}^{n}\right)$. Then

$$
\lim _{k \rightarrow \infty}\left\|\Delta^{k} f\right\|_{2}^{1 /(2 k)}=\sigma_{\hat{f}}
$$


where

$$
\sigma_{\hat{f}}=\sup \{|\vec{\xi}|: \vec{\xi} \in \operatorname{supp} \hat{f}\}
$$

and

$$
\Delta=\frac{\partial^{2}}{\partial u_{1}^{2}}+\cdots+\frac{\partial^{2}}{\partial u_{n}^{2}} .
$$

Furthermore,

$$
\widehat{\Delta^{k}} f(\vec{\xi})=(-1)^{k}|\vec{\xi}|^{2 k} \hat{f}(\vec{\xi}),
$$

where $|\vec{\xi}|=\sqrt{\xi_{1}^{2}+\cdots+\xi_{n}^{2}}$.

(2) The following statement is called the Paley-Wiener-type theorem for the Fourier transform on $\mathbb{R}^{n}$. A function $f$, square integrable on $\mathbb{R}^{n}$, is the restriction on $\mathbb{R}^{n}$ of an entire function of exponential type if, and only if $\Delta^{k} f$ belong to $L_{2}\left(\mathbb{R}^{n}\right)$ for all positive integers $k$, and the limit (2.1) is finite.

Proof. See the proof of Lemma 2.2 in [11].

Let $\mathcal{S}\left(\mathbb{R}^{n}\right)$ be the Schwartz space of infinitely differentiable functions $f(\vec{u})$ decaying at infinity together with all its derivatives faster than any polynomial of $|\vec{u}|^{-1}$.

Note that the Fourier transform is an isomorphism on the Schwartz space $\mathcal{S}\left(\mathbb{R}^{n}\right)$. Also, $\Delta^{k} f$ and $\widehat{\Delta^{k} f}$ are elements of $\mathcal{S}\left(\mathbb{R}^{n}\right)$ for all $k=1,2, \ldots$

For $v \in L_{2}[0, T]$ and $x \in C_{0}[0, T]$, let $\langle v, x\rangle$ denote the PWZ stochastic integral. One can show that for each $v \in L_{2}[0, T],\langle v, x\rangle$ exists for a.e. $x \in$ $C_{0}[0, T]$ and if $v \in L_{2}[0, T]$ is a function of bounded variation on $[0, T],\langle v, x\rangle$ equals the Riemann-Stieltjes integral $\int_{0}^{T} v(t) d x(t)$ for s-a.e. $x \in C_{0}[0, T]$. Also, $\langle v, x\rangle$ has the expected linearity property. Furthermore, $\langle v, x\rangle$ is a Gaussian process with mean 0 and variance $\|v\|_{2}^{2}$. For a more detailed study of the PWZ stochastic integral, see $[2,3,7,8,9]$.

Let $\left\{\alpha_{1}, \alpha_{2}, \ldots\right\}$ be any complete orthonormal set of functions in $L_{2}[0, T]$ and let $h: \mathbb{R}^{n} \rightarrow \mathbb{R}$ be Borel measurable. The following well-known integration formula is used several times in this paper.

$$
\begin{aligned}
& \int_{C_{0}[0, T]} h\left(\left\langle\alpha_{1}, x\right\rangle, \ldots,\left\langle\alpha_{n}, x\right\rangle\right) d m(x) \\
= & \left(\frac{1}{2 \pi}\right)^{\frac{n}{2}} \int_{\mathbb{R}^{n}} h(\vec{v}) \exp \left\{-\frac{|\vec{v}|^{2}}{2}\right\} d \vec{v}
\end{aligned}
$$

in the sense that if either side of (2.3) exists, both sides exist and equality holds.

To simply expressions, we use the following notation

$$
f\left(\left\langle\alpha_{1}, x\right\rangle, \ldots,\left\langle\alpha_{n}, x\right\rangle\right) \equiv f(\langle\vec{\alpha}, x\rangle) .
$$

Now we introduce the Fourier-type functionals defined on $C_{0}[0, T]$. 
Definition 2.3. Let $\left\{\alpha_{1}, \alpha_{2}, \ldots\right\}$ be any complete orthonormal set of functions in $L_{2}[0, T]$. For $f \in \mathcal{S}\left(\mathbb{R}^{n}\right)$, let

$$
\Delta^{k} F(x)=\left(\Delta^{k} f\right)(\langle\vec{\alpha}, x\rangle), \quad k=0,1, \ldots,
$$

and

$$
\widehat{\Delta^{k} F}(x)=\widehat{\Delta^{k}} f(\langle\vec{\alpha}, x\rangle), \quad k=0,1, \ldots
$$

The functionals in (2.4) and (2.5) are called the Fourier-type functionals defined on Wiener space $C_{0}[0, T]$.

Example 2.4. For $n=2$, let $f\left(u_{1}, u_{2}\right)=\exp \left\{-\frac{1}{2} u_{1}^{2}-u_{2}^{2}\right\}$. Then we can easily show that $f$ is in $\mathcal{S}\left(\mathbb{R}^{2}\right), \hat{f}\left(\xi_{1}, \xi_{2}\right)=\frac{1}{\sqrt{2}} \exp \left\{-\frac{1}{2} \xi_{1}^{2}-\frac{1}{4} \xi_{2}^{2}\right\}$ and $\Delta f\left(u_{1}, u_{2}\right)=$ $\left(-3+u_{1}^{2}+4 u_{2}^{2}\right) \exp \left\{-\frac{1}{2} u_{1}^{2}-u_{2}^{2}\right\}$, and hence

$$
\begin{aligned}
\hat{f}(\langle\vec{\alpha}, x\rangle) & =\hat{f}\left(\left\langle\alpha_{1}, x\right\rangle,\left\langle\alpha_{2}, x\right\rangle\right) \\
& =\frac{1}{\sqrt{2}} \exp \left\{-\frac{1}{2}\left\langle\alpha_{1}, x\right\rangle^{2}-\frac{1}{4}\left\langle\alpha_{2}, x\right\rangle^{2}\right\},
\end{aligned}
$$

and

$$
\begin{aligned}
(\Delta f)(\langle\vec{\alpha}, x\rangle) & =(\Delta f)\left(\left\langle\alpha_{1}, x\right\rangle,\left\langle\alpha_{2}, x\right\rangle\right) \\
& =\left(-3+\left\langle\alpha_{1}, x\right\rangle^{2}+4\left\langle\alpha_{2}, x\right\rangle^{2}\right) \exp \left\{-\frac{1}{2}\left\langle\alpha_{1}, x\right\rangle^{2}-\left\langle\alpha_{2}, x\right\rangle^{2}\right\} .
\end{aligned}
$$

Remark 2.5. (1) Using equation (2.2), the Fourier-type functional $\widehat{\Delta^{k} F}$ given by equation (2.5) can be expressed by

$$
\begin{aligned}
\widehat{\Delta^{k} F}(x) & =\widehat{\Delta^{k}} f(\langle\vec{\alpha}, x\rangle) \\
& =(-1)^{k}|\langle\vec{\alpha}, x\rangle|^{2 k} \hat{f}(\langle\vec{\alpha}, x\rangle) \\
& \equiv H_{k}(x) \widehat{F}(x),
\end{aligned}
$$

where

$$
H_{k}(x)=(-1)^{k}|\langle\vec{\alpha}, x\rangle|^{2 k} .
$$

(2) Note that $\Delta^{0} F(x)=F(x), \widehat{\Delta^{0} F}(x)=\widehat{F}(x)$ and $H_{0}(x)=1$.

\section{Some properties of the Fourier-type functionals}

In this section we establish some properties of the Fourier-type functionals given by equations (2.4) and (2.5).

The following lemma plays a key role in the main results of this paper.

Lemma 3.1. Let $f$ and $\Delta^{k} f$ be as in Lemma 2.2 with $\|f\|_{2} \neq 0$. Let $\widehat{\Delta^{k} F}$ be given by equation (2.5). Then

$$
\lim _{k \rightarrow \infty}\left[\frac{1}{\|f\|_{2}^{2}} \int_{\operatorname{supp} \hat{f}}|\vec{u}|^{4 k}|\hat{f}(\vec{u})|^{2} d \vec{u}\right]^{1 /(4 k)}=\sigma_{\hat{f}},
$$


where $\sigma_{\hat{f}}$ is as in Lemma 2.2. Furthermore,

$$
\left\|\widehat{\Delta^{k} F}\right\|_{2} \leq\left\|\widehat{\Delta^{k} f}\right\|_{2}
$$

for all $k=0,1, \ldots$.

Proof. See the proof of equation (3.1) in [11]. For all $k=0,1, \ldots$,

$$
\begin{aligned}
\left\|\widehat{\Delta^{k} F}\right\|_{2}^{2} & =\int_{C_{0}[0, T]}\left|\widehat{\Delta^{k}} f(\langle\vec{\alpha}, x\rangle)\right|^{2} d m(x) \\
& =\left(\frac{1}{2 \pi}\right)^{\frac{n}{2}} \int_{\mathbb{R}^{n}}\left|\widehat{\Delta^{k}} f(\vec{u})\right|^{2} \exp \left\{-\frac{|\vec{u}|^{2}}{2}\right\} d \vec{u} \\
& \leq \int_{\mathbb{R}^{n}}\left|\widehat{\Delta^{k}} f(\vec{u})\right|^{2} d \vec{u}=\left\|\widehat{\Delta^{k}} f\right\|_{2}^{2},
\end{aligned}
$$

which establishes inequality (3.2).

The following theorem is one of the main results in this paper.

Theorem 3.2. Let $\Delta^{k} F$ and $\widehat{\Delta^{k} F}$ be given by equations (2.4) and (2.5). Then

$$
\int_{C_{0}[0, T]} \Delta^{k} F(x) d m(x)=\int_{C_{0}[0, T]} \widehat{\Delta^{k} F}(x) d m(x)
$$

for all $k=1,2, \ldots$. Furthermore, both sides of the expression in equation (3.3) are equal to the expression

$$
\int_{C_{0}[0, T]} H_{k}(x) \widehat{F}(x) d m(x)
$$

for all $k=1,2, \ldots$, where $H_{k}$ is given by equation (2.7).

Proof. Using formula (2.3), we easily obtain that

$$
\int_{C_{0}[0, T]} \Delta^{k} F(x) d m(x)=\left(\frac{1}{2 \pi}\right)^{\frac{n}{2}} \int_{\mathbb{R}^{n}}\left(\Delta^{k} f\right)(\vec{v}) \exp \left\{-\frac{|\vec{v}|^{2}}{2}\right\} d \vec{v} .
$$

While, using formula (2.3) and equation (1.1),

$$
\begin{aligned}
& \int_{C_{0}[0, T]} \widehat{\Delta^{k} F}(x) d m(x) \\
= & \left(\frac{1}{2 \pi}\right)^{\frac{n}{2}} \int_{\mathbb{R}^{n}} \widehat{\Delta^{k}} f(\vec{\xi}) \exp \left\{-\frac{|\vec{\xi}|^{2}}{2}\right\} d \vec{\xi} \\
= & \left(\frac{1}{2 \pi}\right)^{n} \int_{\mathbb{R}^{n}} \int_{\mathbb{R}^{n}}\left(\Delta^{k} f\right)(\vec{v}) \exp \{i \vec{v} \cdot \vec{\xi}\} d \vec{v} \exp \left\{-\frac{|\vec{\xi}|^{2}}{2}\right\} d \vec{\xi} \\
= & \left(\frac{1}{2 \pi}\right)^{n} \int_{\mathbb{R}^{n}}\left(\Delta^{k} f\right)(\vec{v}) \int_{\mathbb{R}^{n}} \exp \left\{-\frac{|\vec{\xi}-i \vec{v}|^{2}}{2}\right\} d \vec{\xi} \exp \left\{-\frac{|\vec{v}|^{2}}{2}\right\} d \vec{v} \\
= & \left(\frac{1}{2 \pi}\right)^{\frac{n}{2}} \int_{\mathbb{R}^{n}}\left(\Delta^{k} f\right)(\vec{v}) \exp \left\{-\frac{|\vec{v}|^{2}}{2}\right\} d \vec{v} .
\end{aligned}
$$


The last equality in equation (3.5) follows immediately from the following integration formula

$$
\left(\frac{1}{2 \pi}\right)^{\frac{1}{2}} \int_{\mathbb{R}} \exp \left\{-\frac{(v-i m)^{2}}{2}\right\} d v=1
$$

for all $m \in \mathbb{R}$. Hence we have obtained equation (3.3). Furthermore, using equation (2.6), we can establish expression (3.4).

The following corollary is a special case of Theorem 3.2 when $k=0$.

Corollary 3.3. We have

$$
\int_{C_{0}[0, T]} F(x) d m(x)=\int_{C_{0}[0, T]} \widehat{F}(x) d m(x) .
$$

Remark 3.4. Using Theorem 3.2, together with equations (2.2) and (2.3), we can establish the following equation

$$
\begin{aligned}
\int_{\mathbb{R}^{n}}\left(\Delta^{k} f\right)(\vec{v}) \exp \left\{-\frac{|\vec{v}|^{2}}{2}\right\} d \vec{v} & =\int_{\mathbb{R}^{n}} \widehat{\left(\Delta^{k} f\right)}(\vec{v}) \exp \left\{-\frac{|\vec{v}|^{2}}{2}\right\} d \vec{v} \\
& =(-1)^{k} \int_{\mathbb{R}^{n}}|\vec{v}|^{2 k} \widehat{f}(\vec{v}) \exp \left\{-\frac{|\vec{v}|^{2}}{2}\right\} d \vec{v}
\end{aligned}
$$

for all $k=1,2, \ldots$ Equation (3.6) can be obtained also from the Parseval formula for the Fourier transform

$$
\int_{\mathbb{R}^{n}} g(\vec{u}-\vec{v}) f(\vec{v}) d \vec{v}=\int_{\mathbb{R}^{n}} \hat{g}(\vec{\xi}) \hat{f}(\vec{\xi}) \exp \{i \vec{u} \cdot \vec{\xi}\} d \vec{\xi}
$$

with

$$
g(\vec{v})=\exp \left\{-\frac{|\vec{v}|^{2}}{2}\right\}
$$

and $\vec{u}=\overrightarrow{0}$.

The following theorem is one of main results in this paper.

Theorem 3.5. Let $\widehat{\Delta^{k} F}$ be given by equation (2.5). Then

$$
\lim _{k \rightarrow \infty}\left\|\widehat{\Delta^{k} F}\right\|_{2}^{1 /(2 k)}=\sigma_{\hat{f}}
$$

where $\sigma_{\hat{f}}$ is as in Lemma 2.2.

Proof. Inequality (3.2) yields that $\widehat{\Delta^{k} F}$ is an element of $L_{2}\left(C_{0}[0, T]\right)$ for all $k=0,1, \ldots$. Furthermore, using equation (2.2),

$$
\begin{aligned}
\left\|\widehat{\Delta^{k} F}\right\|_{2}^{2} & \leq\left\|\widehat{\Delta^{k} f}\right\|_{2}^{2}=\int_{\mathbb{R}^{n}}\left|\widehat{\Delta^{k}} f(\vec{\xi})\right|^{2} d \vec{\xi} \\
& =\int_{\mathbb{R}^{n}}|\vec{\xi}|^{4 k}|\hat{f}(\vec{\xi})|^{2} d \vec{\xi}=\int_{\operatorname{supp} \hat{f}}|\vec{\xi}|^{4 k}|\hat{f}(\vec{\xi})|^{2} d \vec{\xi}
\end{aligned}
$$


From equation (3.8) we obtain

$$
\begin{aligned}
\left\|\widehat{\Delta^{k} F}\right\|_{2}^{1 /(2 k)} & \leq\left[\int_{\operatorname{supp} \hat{f}}|\vec{\xi}|^{4 k}|\hat{f}(\vec{\xi})|^{2} d \vec{\xi}\right]^{1 /(4 k)} \\
& =\|f\|_{2}^{1 /(2 k)}\left[\frac{1}{\|f\|_{2}^{2}} \int_{\operatorname{supp} \hat{f}}|\vec{\xi}|^{4 k}|\hat{f}(\vec{\xi})|^{2} d \vec{\xi}\right]^{1 /(4 k)}
\end{aligned}
$$

for all $k=1,2, \ldots$. Using equation (3.1), the last expression in equation (3.9) goes to $\sigma_{\hat{f}}$ as $k \rightarrow \infty$. Hence we have established inequality

$$
\lim _{k \rightarrow \infty}\left\|\widehat{\Delta^{k} F}\right\|_{2}^{1 /(2 k)} \leq \sigma_{\hat{f}}
$$

On the other hand, note that for any $\varepsilon>0$,

$$
\begin{aligned}
\left\|\widehat{\Delta^{k} F}\right\|_{2}^{2} & =\left(\frac{1}{2 \pi}\right)^{\frac{n}{2}} \int_{\mathbb{R}^{n}}|\vec{\xi}|^{4 k}|\hat{f}(\vec{\xi})|^{2} \exp \left\{-\frac{|\vec{\xi}|^{2}}{2}\right\} d \vec{\xi} \\
& \geq\left(\frac{1}{2 \pi}\right)^{\frac{n}{2}} \int_{W}|\vec{\xi}|^{4 k}|\hat{f}(\vec{\xi})|^{2} \exp \left\{-\frac{|\vec{\xi}|^{2}}{2}\right\} d \vec{\xi} \\
& \geq\left(\sigma_{\hat{f}}-\varepsilon\right)^{4 k}\left(\frac{1}{2 \pi}\right)^{\frac{n}{2}} \int_{W}|\hat{f}(\vec{\xi})|^{2} \exp \left\{-\frac{|\vec{\xi}|^{2}}{2}\right\} d \vec{\xi} \\
& \geq\left(\sigma_{\hat{f}}-\varepsilon\right)^{4 k} C,
\end{aligned}
$$

where $W=\left\{\vec{\xi} \in \mathbb{R}^{n}:|\vec{\xi}| \geq \sigma_{\hat{f}}-\varepsilon\right\}$ and

$$
C=\left(\frac{1}{2 \pi}\right)^{\frac{n}{2}} \int_{W}|\hat{f}(\vec{\xi})|^{2} \exp \left\{-\frac{|\vec{\xi}|^{2}}{2}\right\} d \vec{\xi}>0
$$

Inequality (3.11) tells us that

$$
\left\|\widehat{\Delta^{k} F}\right\|_{2}^{1 /(2 k)} \geq\left(\sigma_{\hat{f}}-\varepsilon\right) C^{1 /(4 k)}
$$

for all $k=1,2, \ldots$. Since $C$ is a positive real number, the last expression (3.12) goes to $\left(\sigma_{\hat{f}}-\varepsilon\right)$ as $k \rightarrow \infty$. Consequently,

$$
\lim _{k \rightarrow \infty}\left\|\widehat{\Delta^{k} F}\right\|_{2}^{1 /(2 k)} \geq \sigma_{\hat{f}}
$$

and hence inequalities (3.10) and (3.13) establish equation (3.7).

In the following corollary we establish a property of the Fourier-type functionals $\Delta^{k} F$ given by equation (2.4).

Corollary 3.6. Let $\Delta^{k} F$ be given by equation (2.4). Then

$$
\lim _{k \rightarrow \infty}\left\|\Delta^{k} F\right\|_{2}^{1 /(2 k)} \leq \sigma_{\hat{f}}
$$

where $\sigma_{\hat{f}}$ is as in Lemma 2.2. 
Proof. First we note that we can easily have an inequality $\left\|\Delta^{k} F\right\|_{2} \leq\left\|\Delta^{k} f\right\|_{2}$ and hence

$$
\left\|\Delta^{k} F\right\|_{2}^{1 /(2 k)} \leq\left\|\Delta^{k} f\right\|_{2}^{1 /(2 k)}
$$

for all $k=1,2, \ldots$ Using equation (2.1), the right-hand side of inequality (3.15) goes to $\sigma_{\hat{f}}$ as $k \rightarrow \infty$. Hence we have established inequality (3.14).

\section{Integral transform of the Fourier-type functionals}

In this section we establish integral transform of the Fourier-type functionals which also can be expressed by other Fourier-type functional.

For each $k=0,1, \ldots$, let $f \in \mathcal{S}\left(\mathbb{R}^{n}\right)$,

$$
g_{k}(\vec{s})=\Delta^{k} f(\vec{s}) \exp \left\{-\frac{\gamma^{2}|\vec{s}|^{2}}{2}\right\}
$$

and

$$
m_{k}(\vec{v})=\left(\frac{1}{2 \pi}\right)^{\frac{n}{2}} \int_{\mathbb{R}^{n}} \Delta^{k} f(\gamma \vec{u}+\beta \vec{v}) \exp \left\{-\frac{|\vec{u}|^{2}}{2}\right\} d \vec{u} .
$$

Then $g_{k}$ and $m_{k}$ are in $\mathcal{S}\left(\mathbb{R}^{n}\right)$ because $\Delta^{k} f$ are in $\mathcal{S}\left(\mathbb{R}^{n}\right)$, and hence their Fourier transforms always exist and belong to $\mathcal{S}\left(\mathbb{R}^{n}\right)$ for all $k=0,1, \ldots$ and $\gamma, \beta \in \mathbb{R}$.

The following theorem is one of the main results in this paper.

Theorem 4.1. Let $\gamma$ and $\beta$ be any nonzero real numbers. Let $\widehat{\Delta^{k} F}$ be given by equation (2.5). Then for any $y \in C_{0}[0, T]$, the integral transform of the Fourier-type functional $\widehat{\Delta^{k} F}$ exists and is given by the formula

$$
\mathcal{F}_{\gamma, \beta}\left(\widehat{\Delta^{k} F}\right)(y)=\widehat{G_{k}}(\beta y),
$$

where

$$
G_{k}(y)=g_{k}(\langle\vec{\alpha}, y\rangle)
$$

and $g_{k}$ is given by equation (4.1).

Proof. For each $j=1,2, \ldots, n$, let $v_{j}=\left\langle\alpha_{j}, y\right\rangle$. Then for any $y \in C_{0}[0, T]$,

$$
\begin{aligned}
& \mathcal{F}_{\gamma, \beta}\left(\widehat{\Delta^{k} F}\right)(y) \\
= & \int_{C_{0}[0, T]} \widehat{\Delta^{k} F}(\gamma x+\beta y) d m(x) \\
= & \int_{C_{0}[0, T]} \widehat{\Delta^{k}} f(\gamma\langle\vec{\alpha}, x\rangle+\beta\langle\vec{\alpha}, y\rangle) d m(x) \\
= & \left(\frac{1}{2 \pi}\right)^{\frac{n}{2}} \int_{\mathbb{R}^{n}} \widehat{\Delta^{k}} f(\gamma \vec{\xi}+\beta \vec{v}) \exp \left\{-\frac{|\vec{\xi}|^{2}}{2}\right\} d \vec{\xi} .
\end{aligned}
$$


Now, for each $j=1,2, \ldots, n$, let $\gamma \xi_{j}+\beta v_{j}=\xi_{j}^{\prime}$. Then substituting into equation (4.4) (and then suppressing the primes) gives us the followings

$$
\begin{aligned}
& \mathcal{F}_{\gamma, \beta}\left(\widehat{\Delta^{k} F}\right)(y) \\
= & \left(\frac{1}{2 \pi \gamma^{2}}\right)^{\frac{n}{2}} \int_{\mathbb{R}^{n}} \widehat{\Delta^{k}} f(\vec{\xi}) \exp \left\{-\frac{|\vec{\xi}-\beta \vec{v}|^{2}}{2 \gamma^{2}}\right\} d \vec{\xi} \\
= & \left(\frac{1}{2 \pi \gamma^{2}}\right)^{\frac{n}{2}}\left(\frac{1}{2 \pi}\right)^{\frac{n}{2}} \int_{\mathbb{R}^{n}} \int_{\mathbb{R}^{n}} \Delta^{k} f(\vec{s}) \exp \{i \vec{s} \cdot \vec{\xi}\} d \vec{s} \exp \left\{-\frac{|\vec{\xi}-\beta \vec{v}|^{2}}{2 \gamma^{2}}\right\} d \vec{\xi} \\
= & \left(\frac{1}{2 \pi \gamma^{2}}\right)^{\frac{n}{2}}\left(\frac{1}{2 \pi}\right)^{\frac{n}{2}} \int_{\mathbb{R}^{n}} \Delta^{k} f(\vec{s}) \int_{\mathbb{R}^{n}} \exp \left\{-\frac{|\vec{\xi}-\beta \vec{v}|^{2}}{2 \gamma^{2}}+i \vec{s} \cdot \vec{\xi}\right\} d \vec{\xi} d \vec{s} \\
= & \left(\frac{1}{2 \pi \gamma^{2}}\right)^{\frac{n}{2}}\left(\frac{1}{2 \pi}\right)^{\frac{n}{2}} \int_{\mathbb{R}^{n}} \Delta^{k} f(\vec{s}) \exp \{i \beta \vec{v} \cdot \vec{s}\} \exp \left\{-\frac{\gamma^{2}|\vec{s}|^{2}}{2}\right\} \\
& \times \int_{\mathbb{R}^{n}} \exp \left\{-\frac{\left|\vec{\xi}-\left(\beta \vec{v}+i \gamma^{2} \vec{s}\right)\right|^{2}}{2 \gamma^{2}}\right\} d \vec{\xi} d \vec{s} \\
= & \left(\frac{1}{2 \pi}\right)^{\frac{n}{2}} \int_{\mathbb{R}^{n}} \Delta^{k} f(\vec{s}) \exp \{i \beta \vec{v} \cdot \vec{s}\} \exp \left\{-\frac{\gamma^{2}|\vec{s}|^{2}}{2}\right\} d \vec{s} \\
= & \left(\frac{1}{2 \pi}\right)^{\frac{n}{2}} \int_{\mathbb{R}^{n}} g_{k}(\vec{s}) \exp \{i \beta \vec{v} \cdot \vec{s}\} d \vec{s} .
\end{aligned}
$$

Using equations (1.1), (2.5) and (4.1), the last expression in equation (4.5) equals to the Fourier-type functional $\widehat{G_{k}}(\beta y)=\widehat{g_{k}}(\beta\langle\vec{\alpha}, y\rangle)$. Hence we have established equation (4.3).

Remark 4.2. For all nonzero complex numbers $\gamma$ and $\beta$ with $\operatorname{Re}\left(\gamma^{2}\right)>0$, the function $g_{k}(\vec{s})$ defined by (4.1) is still in $\mathcal{S}\left(\mathbb{R}^{n}\right)$, moreover, $\widehat{G}_{k}(\beta y)$ exists and analytic in $\beta$. Hence, the equation (4.3) still holds by analytic continuation.

The following corollary follows immediately from Theorem 4.1 by letting $\beta=1$ or $k=0$.

Corollary 4.3. When $\beta=1, \mathcal{F}_{\gamma, 1}\left(\widehat{\Delta^{k} F}\right)(y)=\widehat{G_{k}}(y)$, and when $k=0$, $\mathcal{F}_{\gamma, \beta}(\widehat{F})(y)=\widehat{G_{0}}(\beta y)$, where

$$
G_{0}(y)=g_{0}(\langle\vec{\alpha}, y\rangle)
$$

and

$$
g_{0}(\vec{s})=f(\vec{s}) \exp \left\{-\frac{\gamma^{2}|\vec{s}|^{2}}{2}\right\} .
$$

The following theorem is the last main result in this paper.

Theorem 4.4. Let $\gamma$ and $\beta$ be any nonzero complex numbers. Let $\Delta^{k} F$ be given by equation (2.4). Then for any $y \in C_{0}[0, T]$, the integral transform of 
the Fourier-type functional $\Delta^{k} F$ exists and is given by the formula

$$
\mathcal{F}_{\gamma, \beta}\left(\Delta^{k} F\right)(y)=M_{k}(y),
$$

where

$$
M_{k}(y)=m_{k}(\langle\vec{\alpha}, y\rangle)
$$

and $m_{k}$ is given by equation (4.2).

Proof. For each $j=1,2, \ldots, n$, let $v_{j}=\left\langle\alpha_{j}, y\right\rangle$. Then using equation (2.3) it follows that for any $y \in C_{0}[0, T]$,

$$
\begin{aligned}
& \mathcal{F}_{\gamma, \beta}\left(\Delta^{k} F\right)(y) \\
= & \int_{C_{0}[0, T]} \Delta^{k} F(\gamma x+\beta y) d m(x) \\
= & \int_{C_{0}[0, T]}\left(\Delta^{k} f\right)(\gamma\langle\vec{\alpha}, x\rangle+\beta\langle\vec{\alpha}, y\rangle) d m(x) \\
= & \left(\frac{1}{2 \pi}\right)^{\frac{n}{2}} \int_{\mathbb{R}^{n}} \Delta^{k} f(\gamma \vec{u}+\beta \vec{v}) \exp \left\{-\frac{|\vec{u}|^{2}}{2}\right\} d \vec{u} .
\end{aligned}
$$

Hence we have established equation (4.6).

The following corollary follows immediately from Theorem 4.4 by letting $k=0$.

Corollary 4.5. When $k=0, \mathcal{F}_{\gamma, \beta}(F)(y)=M_{0}(y)=m_{0}(\langle\vec{\alpha}, y\rangle)$, where

$$
m_{0}(\vec{v})=\left(\frac{1}{2 \pi}\right)^{\frac{n}{2}} \int_{\mathbb{R}^{n}} f(\gamma \vec{u}+\beta \vec{v}) \exp \left\{-\frac{|\vec{u}|^{2}}{2}\right\} d \vec{u} .
$$

\section{References}

[1] H. H. Bang, Functions with bounded spectrum, Trans. Amer. Math. Soc. 347 (1995), no. 3, 1067-1995.

[2] S. J. Chang, H. S. Chung, and D. Skoug, Convolution products, integral transforms and inverse integral transforms of functionals in $L_{2}\left(C_{0}[0, T]\right)$, Integral Transforms Spec. Funct. 21 (2010), no. 1-2, 143-151.

[3] H. S. Chung and V. K. Tuan, Generalized integral transforms and convolution products on function space, Integral Transforms Spec. Funct. 22 (2011), no. 8, 573-586.

[4] G. W. Johnson and D. L. Skoug, Scale-invariant measurability in Wiener space, Pacific J. Math. 83 (1979), no. 1, 157-176.

[5] B. S. Kim and D. Skoug, Integral transforms of functionals in $L_{2}\left(C_{0}[0, T]\right)$, Rocky Mountain J. Math. 33 (2003), no. 4, 1379-1393.

[6] Y. J. Lee, Integral transforms of analytic functions on abstract Wiener spaces, J. Funct. Anal. 47 (1982), no. 2, 153-164.

[7] R. E. A. C. Paley, N. Wiener and A. Zygmund, Notes on random functions, Math. Z. 37 (1933), no. 1, 647-688.

[8] C. Park, A generalized Paley-Wiener-Zygmund integrals and applications, Proc. Amer. Math. Soc. 23 (1969), 388-400.

[9] C. Park and D. Skoug, A note on Paley-Wiener-Zygmund stochastic integrals, Proc. Amer. Math. Soc. 103 (1988), no. 2, 591-601. 
[10] E. M. Stein and G. Weiss, Introduction to Fourier Analysis on Euclidean Spaces, Princeton Univ. Press, Princeton, 1971.

[11] V. K. Tuan, Paley-Wiener type theorems, Fract. Calc. Appl. Anal. 2 (1999), no. 2, 135-143.

Hyun Soo Chung

Department of Mathematics

DANKOOK UNIVERSITY

Cheonan 330-714, Korea

AND

Department of Mathematics

University of West Georgia

Carrollton 30118, USA

E-mail address: hschung@dankook.ac.kr, hchung@westga.edu

Vu Kim Tuan

Department of Mathematics

University of WeSt GeORgia

CARrollton 30118, USA

E-mail address: vu@westga.edu 